Author Posting. (c) Taylor \& Francis Group, LLC, 2011.

This is the author's version of the work. It is posted here by permission of Taylor \& Francis Group, LLC for personal use, not for redistribution.

The definitive version was published in Communications in Algebra, Volume 39 Issue 1, January 2011.

doi:10.1080/00927870903428254 (http://dx.doi.org/10.1080/00927870903428254)

\title{
SOME ORTHODOX MONOIDS WITH ASSOCIATE INVERSE SUBSEMIGROUPS
}

\author{
B. BILLHARDT, E. GIRALDES, P. MARQUES-SMITH, P. MENDES MARTINS
}

\begin{abstract}
By an associate inverse subsemigroup of a regular semigroup $S$ we mean a subsemigroup $T$ of $S$ containing a least associate of each $x \in S$, in relation to the natural partial order $\leq_{S}$ in $S$. In this paper we investigate a class of orthodox monoids with an associate inverse subsemigroup and obtain a known description of uniquely unit regular orthodox semigroups as a corollary. Also, by considering a more general situation, we identify the homomorphic image of a kind of semidirect product of a band with identity by an inverse monoid, thus extending a known result for unit regular orthodox semigroups.
\end{abstract}

\section{INTRODUCTION}

Extending the concept of associate subgroup of a semigroup, first presented in [4], the authors introduced in [3] the notion of associate inverse subsemigroup of a regular semigroup $S$. This is a (necessarily inverse) subsemigroup $S^{*}$ of $S$ containing a least associate of each $x \in S, x^{*}$, say, in relation to the natural partial order of $S$. The main result of [3] is a description of a regular semigroup with associate inverse subsemigroups satisfying two natural conditions.

A monoid $S$ is said to be [uniquely] unit regular if for every $x \in S$ there is a [unique] unit $u \in S$ such that $x=x u x$. This is an idea that has come to semigroups from the theory of rings [8]. Examples of unit regular semigroups include the full transformation semigroup on a finite set and the semigroup of endomorphisms of a finite-dimensional vector space. In [5] the authors determined the structure of orthodox semigroups that are uniquely unit regular. The structure that they obtained is in terms of the group of units and the band of idempotents. Neither of the examples cited above is uniquely unit regular. However, this class of semigroups coincides with a particular class of $F$-regular

2000 Mathematics Subject Classification. Primary 20M10.

Research supported by the Portuguese Foundation for Science and Technology (FCT) through the Research Centre CMAT.

Corresponding Author: P. Marques-Smith. 
semigroups, i.e., semigroups for which there exists a group congruence such that every congruence class contains a greatest element with respect to the natural partial order [7]. The problem of describing the structure of an arbitrary unit regular semigroup proved to be difficult to solve. In [10] it is shown that every unit regular orthodox semigroup is an idempotent-separating homomorphic image of a uniquely unit orthodox semigroup. In this paper, following the work in [3], we investigate the class of some orthodox monoids with an associate inverse subsemigroup and extend the main results of [5] and [10].

In section 2 we establish the structure of orthodox monoids $S$ with an associate inverse subsemigroup $S^{*}$, satisfying:

$$
\begin{aligned}
& \text { (A1) } \forall s, t \in S, \quad(s t)^{*}=t^{*} s^{*} ; \\
& \text { (A2) } \forall e \in E(S) \forall f^{*} \in E\left(S^{*}\right), \quad e f^{*}=f^{*} e
\end{aligned}
$$

and obtain Blyth and McFadden's description of uniquely unit orthodox semigroups as a corollary. In section 3, we consider a more general situation and show that the orthodox monoids $S$ with an inverse subsemigroup $V$, containing an associate of each element and satisfying $e a=a e$ for all $e \in E(S)$ and all $a \in E(V)$, are exactly the homomorphic images of a $\lambda$-semidirect product of a band with identity by an inverse monoid. This extends McFadden's result for unit regular orthodox semigroups.

For standard notation in semigroup theory, we refer the reader to the books of Grillet [9] and Petrich [11].

\section{RESTRICTED SEMIDIRECT PRODUCTS}

In this section we describe the class of orthodox monoids $S$ with an associate inverse subsemigroup $S^{*}$ satisfying conditions

$$
\begin{aligned}
& \text { (A1) } \forall s, t \in S, \quad(s t)^{*}=t^{*} s^{*} ; \\
& \text { (A2) } \forall e \in E(S) \forall f^{*} \in E\left(S^{*}\right), \quad e f^{*}=f^{*} e .
\end{aligned}
$$

The description that we provide uses the restricted semidirect product of semigroups.

Let $V$ be an inverse semigroup acting on a band $B$ by endomorphisms on the left. Let $\pi: B \rightarrow E(V)$ be an epimorphism such that, for each $e \in B, v \in V$,

$$
v v^{-1} e=e \Leftrightarrow e \pi \leq v v^{-1} .
$$

Then let $S=\left\{(e, v) \in B \times V: v v^{-1}=e \pi\right\}$.

Lemma 2.1. Let $S$ be the set defined above and $(e, v) \in S$. Then,

$$
v^{-1}(e \pi) v=\left(v^{-1} e\right) \pi .
$$


Proof. First, we notice that, for every $\alpha \in E(V)$, we have

$$
{ }^{\alpha}\left(v^{-1} e\right)=v^{-1} e \Longrightarrow v^{-1}(e \pi) v \leq \alpha .
$$

In fact,

$$
\begin{aligned}
& \alpha\left(v^{-1} e\right)=v^{-1} e \Longrightarrow v \alpha v^{-1} e=v v^{-1} e \\
& \Longrightarrow v \alpha v^{-1} e=e \quad((e, v) \in S) \\
& \Longrightarrow e \pi \leq v \alpha v^{-1} \quad \text { definition of } \pi \\
& \Longrightarrow v^{-1}(e \pi) v \leq v^{-1} v \alpha v^{-1} v \\
& \Longrightarrow v^{-1}(e \pi) v \leq \alpha . \quad V \text { is inverse }
\end{aligned}
$$

Since $\left(v^{-1} e\right) \pi \in E(V)$ and, by definition of $\pi,\left(v^{-1} e\right) \pi\left(v^{-1} e\right)=v^{-1} e$, we have, by (1), that

$$
v^{-1}(e \pi) v \leq\left(v^{-1} e\right) \pi \text {. }
$$

Moreover, again by the fact that $(e, v) \in S$ and by definition of $\pi$, we have

$$
v^{-1}(e \pi) v\left(v^{-1} e\right)=v^{-1}(e \pi)\left({ }^{v v^{-1}} e\right)=v^{-1}\left({ }^{(e \pi)} e\right)=v^{-1} e .
$$

Thus, since $v^{-1}(e \pi) v \in E(V)$, we have

$$
\left(v^{-1} e\right) \pi \leq v^{-1}(e \pi) v
$$

By (2) and (3) equality follows.

We are now in condition to prove the following result.

Theorem 2.2. The subset $S$ of $B \times V$ defined as above is an orthodox semigroup under the following multiplication:

$$
(e, v)(f, w)=\left(e^{v} f, v w\right) .
$$

Proof. Let $(e, v),(f, w) \in S$. From $f \pi=w w^{-1}$, we have

$$
v(f \pi) v^{-1}=v w w^{-1} v^{-1}
$$

and, by Lemma 2.1,

$$
\left({ }^{v} f\right) \pi=v w w^{-1} v^{-1}
$$

Thus, from $e \pi=v v^{-1}$, we have

$$
e \pi\left({ }^{v} f\right) \pi=v v^{-1} v w w^{-1} v^{-1}
$$

that is

$$
\left(e^{v} f\right) \pi=v w w^{-1} v^{-1} .
$$

Since the operation is clearly associative, we conclude that $S$ is a semigroup. Moreover, for every $(e, v) \in S,\left(v^{-1} e, v^{-1}\right) \in A((e, v))$. Finally, $(e, v) \in E(S) \Leftrightarrow v \in E(V)$ and so the semigroup is orthodox. 
The semigroup $S$ is called a restricted semidirect product of $B$ by $V$ and is denoted by $B \otimes V$ (see [1]).

Notice that if $B \otimes V$ is a restricted semidirect product, the mapping $\pi$ of the definition is surjective. In fact, if $e_{v} \in E(V)$, then $e_{v} e_{v}=e_{v}$, and, by definition of $\pi$, there exists $f \in B$ such that $f \pi=e_{v} e_{v}^{-1}=e_{v}$.

The previous theorem allows us to conclude that if $(e, v),(f, w) \in S$, then

$$
v w w^{-1} v^{-1} e^{v w w^{-1}} f=e^{v} f
$$

and so the definition (4) is equivalent to the following one:

$$
(e, v)(f, w)=\left(v w w^{-1} v^{-1} e^{v w w^{-1}} f, v w\right) .
$$

In what follows, we will use both definitions.

Theorem 2.3. Let $S$ be an orthodox semigroup with associate inverse subsemigroup $S^{*}$ satisfying conditions (A1) and (A2). Then $S$ is isomorphic to a restricted semidirect product $E(S) \otimes S^{*}$.

Proof. We show first that $S^{*}$ acts on $E(S)$ via

$$
s^{*} e=s^{*} e s^{* *}, \text { for all } s^{*} \in S^{*} .
$$

Given $e, f \in E(S)$, we have

$$
\begin{aligned}
s^{*} e s^{*} f & =s^{*} e s^{* *} s^{*} f s^{* *}=s^{*} e f s^{* *} s^{*} s^{* *} \\
& =s^{*} e f s^{* *}=s^{*}(e f) .
\end{aligned}
$$

Also, for $s^{*}, t^{*} \in S^{*}$,

$$
s^{*} t^{*} e={ }^{(t s)^{*}} e=(t s)^{*} e(t s)^{* *}=s^{*} t^{*} e t^{* *} s^{* *} .
$$

Hence $S^{*}$ acts on $E(S)$.

We proceed to define a homomorphism satisfying the conditions of the definition of restricted semidirect product. For each $e \in E(S),(e e)^{*}=e^{*}$ and $(e e)^{*}=e^{*} e^{*}$ by (A1), whence $e^{*} \in E\left(S^{*}\right)$. So, the assignment $e \mapsto e^{*}$ is a mapping $\pi$, say, from $E(S)$ into $E\left(S^{*}\right)$. Notice that $\pi$ is a morphism - in fact, for every $e, f \in E(S)$,

$$
(e f) \pi=(e f)^{*}=f^{*} e^{*} .
$$

Since $e^{*}, f^{*} \in E\left(S^{*}\right) \subseteq E(S)$, it follows by (A2) that

$$
(e f) \pi=e^{*} f^{*}=(e \pi)(f \pi) .
$$

Thus $\pi$ is a homomorphism.

Next we show that 
(I) $\forall e \in E(S) \forall s^{*} \in S^{*} \quad s^{*}\left(s^{*}\right)^{-1} e=e \Leftrightarrow e \pi \leq s^{*}\left(s^{*}\right)^{-1}$.

We have, on one hand,

$$
\begin{aligned}
s^{*} s^{* *} e=e & \Leftrightarrow s^{*} s^{* *} e\left(s^{*} s^{* *}\right)^{* *}=e \Leftrightarrow s^{*} s^{* *} e s^{* * *} s^{* * * *}=e \Leftrightarrow s^{*} s^{* *} e s^{*} s^{* *}=e \\
& \Leftrightarrow\left\{\begin{array} { l } 
{ s ^ { * } s ^ { * * } s ^ { * } s ^ { * * } e = e } \\
{ e s ^ { * } s ^ { * * } s ^ { * } s ^ { * * } = e }
\end{array} \Leftrightarrow \left\{\begin{array} { l } 
{ s ^ { * } s ^ { * * } e = e } \\
{ e s ^ { * } s ^ { * * } = e }
\end{array} \Rightarrow \left\{\begin{array}{l}
e^{*} s^{* * *} s^{* *}=e^{*} \\
s^{* * *} s^{* *} e^{*}=e^{*}
\end{array}\right.\right.\right. \\
& \Rightarrow\left\{\begin{array}{l}
e^{*} s^{*} s^{* *}=e^{*} \\
s^{*} s^{* *} e^{*}=e^{*}
\end{array} \Rightarrow e^{*} \leq s^{*} s^{* *} \Leftrightarrow e \pi \leq s^{*}\left(s^{*}\right)^{-1} .\right.
\end{aligned}
$$

Conversely, suppose that $e^{*} \leq s^{*} s^{* *}$. We have

$$
e=e e^{*} e=e e^{*} s^{*} s^{* *} e=e e^{*} e s^{*} s^{* *}=e s^{*} s^{* *}
$$

and

$$
e=e e^{*} e=e e^{*} s^{*} s^{* *} e=s^{*} s^{* *} e e^{*} e=s^{*} s^{* *} e .
$$

It follows that $s^{*} s^{* *} e s^{*} s^{* *}=e$ and so $s^{*} s^{* *} e=e$.

(II) $\forall s^{*} \in S^{*} \exists e \in E(S): e \pi=s^{*} s^{* *}$.

Let $s^{*} \in S^{*}$. The element $s^{*} s$ is an idempotent of $S$ since $s=s s^{*} s$. Moreover,

$$
\left(s^{*} s\right) \pi=\left(s^{*} s\right)^{*}=s^{*} s^{* *} .
$$

In view of this, we can define the restricted semidirect product of $E(S)$ by $S^{*}$ with respect to the action and $\pi$ defined above:

$$
E(S) \otimes S^{*}=\left\{\left(e, s^{* *}\right): s^{* *} s^{*}=e^{*}\right\} .
$$

Recall that the multiplication is given by

$$
\begin{aligned}
\left(e, s^{* *}\right)\left(f, t^{* *}\right) & =\left(e^{s^{* *}} f, s^{* *} t^{* *}\right) \\
& =\left(e s^{* *} f s^{*}, s^{* *} t^{* *}\right) .
\end{aligned}
$$

We show that $S$ is isomorphic to the restricted semidirect product $E(S) \otimes S^{*}$.

For each $s \in S$, the element $\left(s s^{*}, s^{* *}\right)$ of $E(S) \otimes S^{*}$ is uniquely determined. So, we can define the mapping $\psi$ as follows:

$$
\begin{aligned}
\psi: S & \rightarrow E(S) \otimes S^{*} \\
s & \mapsto\left(s s^{*}, s^{* *}\right)
\end{aligned}
$$

We show that $\psi$ is a morphism. In fact, for any $s, t \in S$, we have

$$
\begin{aligned}
s \psi \cdot t \psi & =\left(s s^{*}, s^{* *}\right)\left(t t^{*}, t^{* *}\right)=\left(s s^{*} s^{* *} t t^{*} s^{*}, s^{* *} t^{* *}\right) \\
& =\left(s t t^{*} s^{*} s^{* *} s^{*}, s^{* *} t^{* *}\right)=\left(s t t^{*} s^{*}, s^{* *} t^{* *}\right) \\
& =(s t) \psi .
\end{aligned}
$$


Moreover, $\psi$ is injective. Let $s, t \in S$ be such that $s \psi=t \psi$. We have

$$
\begin{aligned}
s \psi=t \psi & \Leftrightarrow\left(s s^{*}, s^{* *}\right)=\left(t t^{*}, t^{* *}\right) \\
& \Rightarrow s s^{*}=t t^{*} \text { and } s^{* *}=t^{* *} \\
& \Rightarrow s=s \cdot s^{*} s^{* *} \cdot s^{*} s=s s^{*} s s^{*} s^{* *}=s s^{*} s^{* *}=t t^{*} t^{* *}=t .
\end{aligned}
$$

We finally show that $\psi$ is surjective. Let $(e, v) \in E(S) \otimes S^{*}$. We show that $(e, v)=(e v) \psi$. We have

$$
\begin{aligned}
(e v) \psi & =\left((e v)(e v)^{*},(e v)^{* *}\right) \\
& =\left(e v v^{*} e^{*}, e^{* *} v^{* *}\right)=\left(e v^{* *} v^{*}, e^{*} v\right) \\
& =\left(e v^{* *} v^{*}, v v^{*} v\right)=(e, v) .
\end{aligned}
$$

Corollary 2.4. An orthodox monoid with an associate inverse subsemigroup satisfying properties (A1) and (A2) is isomorphic to a restricted semidirect product of a band monoid by an inverse monoid.

The converse of Theorem 2.3 does not hold. Consider the following example.

Example 2.1. Let $B=\{x, y, z\}$ be a right zero semigroup and $G=\{u, a\}$ the two element group. Let $G$ be acting on $B$ via ${ }^{g} e=e$, for all $e \in B$ and for all $g \in G$. Clearly, the constant morphism $\pi: B \rightarrow\{u\}$ satisfies, for each $e \in B, g \in G$,

$$
g g^{-1} e=e \Leftrightarrow e \pi \leq g g^{-1}
$$

and, for each $g \in G$, there exists $e \in B$ such that $e \pi=g g^{-1}$.

Let $S=B \times G$. Then $S=\left\{(e, g) \in B \times G: g g^{-1}=e \pi\right\}$. By the definition of restricted semidirect product, $S=B \otimes G$ and $S$ is orthodox (Theorem 2.2). Clearly, $S^{*}=$ $\{(x, u),(y, u)\}$ is an associate inverse subsemigroup of $S$. Also, $E(S)=\{(x, u),(y, u),(z, u)\}$. However, condition (A2) does not hold: in fact, $(y, u) \in E(S),(x, u) \in E\left(S^{*}\right)$ and

$$
(y, u)(x, u)=\left(y^{u} x, u\right)=(x, u) \neq(y, u)=(x, u)(y, u) .
$$

The next theorem shows that the converse of Corollary 2.4 is true.

Theorem 2.5. Let $S=B \otimes V$ be a restricted semidirect product of a band monoid $B$, with identity $\varepsilon$, by an inverse monoid $V$ with identity $1_{V}$. Then $S$ is an orthodox monoid with an associate inverse submonoid $S^{*}=\left\{\left({ }^{v v^{-1}} \varepsilon, v\right): v \in V\right\}$ isomorphic to $V$, satisfying (A1) and (A2).

Proof. Let $\pi: B \rightarrow E(V)$ be the morphism of the definition of restricted semidirect product. We show first that $\left(\varepsilon, 1_{V}\right)$ is the identity of $S$. In order to show that, notice that $\varepsilon \pi=1_{V}$ since $\pi$ is a surjective homomorphism. It follows that $\left(\varepsilon, 1_{V}\right) \in S$ since $\varepsilon \pi=1_{V}\left(=1_{V} 1_{V}^{-1}\right)$. Moreover, for each $f \in B$,

$$
{ }^{1_{V}} f={ }^{1_{V}}(\underset{6}{f \pi} f)={ }^{f \pi} f=f
$$


and so ${ }^{1_{V}} f=f$. We can now show that $\left(\varepsilon, 1_{V}\right)$ is the identity of $S$. Let $(f, w) \in S$. We have

$$
\left(\varepsilon, 1_{V}\right)(f, w)=\left(\varepsilon^{1_{V}} f, 1_{V} w\right)=(\varepsilon f, w)=(f, w)
$$

and

$$
\begin{aligned}
(f, w)\left(\varepsilon, 1_{V}\right) & =\left(f^{w} \varepsilon, w 1_{V}\right)=\left(w^{-1} f w^{-1} w \varepsilon, w\right) \\
& =\left(w w^{-1} w\left(w^{-1} f \varepsilon\right), w\right)=\left(w w^{-1} w w^{-1} f, w\right)=(f, w) .
\end{aligned}
$$

We proceed to show that the set $S^{*}=\left\{\left({ }^{v v^{-1}} \varepsilon, v\right): v \in V\right\}$ is a submonoid of $S(=B \otimes V)$.

(I) $S^{*} \subseteq B \otimes V$.

Let $\left({ }^{v v^{-1}} \varepsilon, v\right) \in S^{*}$. Then $\left({ }^{v v^{-1}} \varepsilon, v\right) \in B \times V$ and we show that $v v^{-1}=\left({ }^{v v^{-1}} \varepsilon\right) \pi$. On one hand, by definition of $\pi$,

$$
{ }^{v v^{-1}}\left({ }^{v v^{-1}} \varepsilon\right)={ }^{v v^{-1}} \varepsilon \Longrightarrow\left({ }^{v v^{-1}} \varepsilon\right) \pi \leq v v^{-1} .
$$

On the other hand, let $e \in B$ be such that $v v^{-1}=e \pi$ (according to the definition of semidirect restricted product). We show that $v v^{-1} \leq\left(v v^{-1} \varepsilon\right) \pi$. In fact,

$$
v v^{-1}\left(v^{-1} \varepsilon\right) \pi=\left({ }^{v v^{-1}} e\right) \pi\left({ }^{v v^{-1}} \varepsilon\right) \pi=\left({ }^{v v^{-1}}(e \varepsilon)\right) \pi=\left({ }^{v v^{-1}} e\right) \pi=v v^{-1} .
$$

Since $v v^{-1},\left({ }^{v v^{-1}} \varepsilon\right) \pi \in E(S)$, we conclude that

$$
v v^{-1} \leq\left(v^{-1} \varepsilon\right) \pi
$$

From (a) and (b) it follows that $v v^{-1}=\left({ }^{v v^{-1}} \varepsilon\right) \pi$. Thus $S^{*} \subseteq B \otimes V$.

(II) Let $\left({ }^{v v^{-1}} \varepsilon, v\right),\left({ }^{w w^{-1}} \varepsilon, w\right) \in S^{*}$. We have

$$
\begin{aligned}
& \left({ }^{v v^{-1}} \varepsilon, v\right)\left({ }^{w w^{-1}} \varepsilon, w\right)=\left({ }^{v w w^{-1} v^{-1}} \varepsilon^{v w w^{-1}} \varepsilon, v w\right) \\
& =\left({ }^{v w w^{-1}}\left(v^{-1} \varepsilon \varepsilon\right), v w\right) \\
& =\left(v w w^{-1} v^{-1} \varepsilon, v w\right) \\
& =\left(v w(v w)^{-1} \varepsilon, v w\right) \in S^{*} .
\end{aligned}
$$

Since $\left(\varepsilon, 1_{V}\right) \in S^{*}$, it follows that $S^{*}$ is a submonoid of $S$.

Next we show that $S^{*} \simeq V$. Let $\varphi: V \rightarrow S^{*}$ be the mapping defined by $v \varphi=\left({ }^{v v^{-1}} \varepsilon, v\right)$ for each $v \in V$. It is clear that $\varphi$ is a bijection. Also, given $v, w \in V$,

$$
\begin{aligned}
& v \varphi \cdot w \varphi=\left({ }^{v v^{-1}} \varepsilon, v\right)\left({ }^{w w^{-1}} \varepsilon, w\right)=\left({ }^{v w w^{-1} v^{-1}} \varepsilon^{v w w^{-1}} \varepsilon, v w\right) \\
& =\left(v w w^{-1}\left(v^{-1} \varepsilon \varepsilon\right), v w\right)=\left(v w w^{-1} v^{-1} \varepsilon, v w\right)=(v w) \varphi .
\end{aligned}
$$

Hence $\varphi$ is an isomorphism and so the monoid $S^{*}$ is inverse.

The monoid $S^{*}$ is also associate. Let $(e, v) \in S$. Since $v \in V$ and $V$ is inverse, $v^{-1}$ is the least associate of $v$. So, if there exists the least associate of $(e, v)$ it must be $\left(v^{-1} v \varepsilon, v^{-1}\right)$. 
We show that $\left(v^{-1} v \varepsilon, v^{-1}\right)$ is an associate of $(e, v)$.

$$
\begin{aligned}
(e, v)\left(v^{-1} v \varepsilon, v^{-1}\right)(e, v) & =\left(e^{v v^{-1} v} \varepsilon, v v^{-1}\right)(e, v) \\
& =\left(e^{v} \varepsilon, v v^{-1}\right)(e, v) \\
& =\left(e^{v} \varepsilon^{v v^{-1}} e, v\right) \\
& =\left(e^{v}\left(\varepsilon^{v^{-1}} e\right), v\right) \\
& =\left(e^{v v^{-1}} e, v\right)=(e, v) .
\end{aligned}
$$

Finally we show that (A1) and (A2) are satisfied.

Let $e, f \in B$ and $v, w \in V$. We have

$$
\begin{aligned}
((e, v)(f, w))^{*} & =\left(e^{v} f, v w\right)^{*}=\left({ }^{(v w)^{-1} v w} \varepsilon,(v w)^{-1}\right) \\
& =\left({ }^{-1} v^{-1} v w \varepsilon, w^{-1} v^{-1}\right)=\left(w^{-1} w \varepsilon, w^{-1}\right)\left({ }^{v^{-1}} \varepsilon, v^{-1}\right) \\
& =(f, w)^{*}(e, v)^{*}
\end{aligned}
$$

Now let $(e, v) \in E(S)$ and $\left({ }^{w w^{-1}} \varepsilon, w\right)^{*} \in E\left(S^{*}\right)$. Then $v, w \in E(V)$. We have

$$
\begin{aligned}
(e, v)\left({ }^{w w^{-1}} \varepsilon, w\right)^{*} & =(e, v)\left({ }^{w} \varepsilon, w\right)=\left({ }^{v w} e^{v w} \varepsilon, v w\right)=\left({ }^{v w}(e \varepsilon), v w\right) \\
& =\left({ }^{w v}(\varepsilon e), w v\right)=\left({ }^{w} \varepsilon, w\right)(e, v)=\left({ }^{w w^{-1}} \varepsilon, w\right)^{*}(e, v)
\end{aligned}
$$

Combining Corollary 2.4 and Theorem 2.5 we obtain

Theorem 2.6. Let $S=B \otimes V$ be a restricted semidirect product of a band monoid $B$ by an inverse monoid $V$. Then $S$ is an orthodox monoid with associate inverse subsemigroup $S^{*}$, satisfying properties
(A1) $\forall s, t \in S, \quad(s t)^{*}=t^{*} s^{*}$;
(A2) $\forall e \in E(S) \forall f^{*} \in E\left(S^{*}\right), \quad e f^{*}=f^{*} e$.

Conversely, every such semigroup $T$ can be constructed this way, where $B=E(T)$ and $V=T^{*}$.

The following example illustrates the previous theorem.

Example 2.2. Let $B$ be the 3 element chain

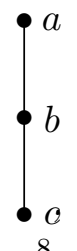


$G=\{u, x\}$ the two element group and $S=B \times G$ be the direct product. Then $E(S)=$ $B \times\{u\}$ and $S$ is clearly orthodox with identity $(a, u)$. Also, $T=\{(a, x),(a, u)\}$ is a subsemigroup of $S$ isomorphic to $G$, where the least associate in $T$ of the elements of $B \times\{u\}$ and of $B \times\{x\}$ is, respectively, $(a, u)$ and $(a, x)$. Easy calculations show that properties (A1) and (A2) are satisfied. The semigroup $S$ is also unit regular.

We observe that Theorem 4 and Theorem 5 of [5] are corollaries of [[7], Theorem 6.4] and of Corollary 2.4 above, respectively. In fact, if $S$ is uniquely unit orthodox then $S$ is an orthodox monoid with an inverse subsemigroup satisfying (A1) and (A2) and so, by Corollary 2.4, $S$ is isomorphic to a restricted semidirect product of a band monoid by an inverse monoid. In this case, the inverse monoid is the group of units and the mapping $\pi$ of the definition is trivial. So the restricted semidirect product is the classical semidirect product. The converse is an immediate consequence of [[7], Theorem 6.4].

The next example shows that orthodox monoids with an inverse semigroup satisfying (A1) and (A2) are not necessarily uniquely unit orthodox.

Example 2.3. Take $\mathcal{I}\left(X_{2}\right)$, the symmetric inverse semigroup on a two element set.

Consider the subsemigroup $V=\{0, x, y, z, w, 1\}$ of $\mathcal{I}\left(X_{2}\right)$, where

$$
x=\left(\begin{array}{l}
1 \\
1
\end{array}\right), y=\left(\begin{array}{l}
2 \\
2
\end{array}\right), z=\left(\begin{array}{l}
1 \\
2
\end{array}\right), w=\left(\begin{array}{l}
2 \\
1
\end{array}\right), 1=i d_{X_{2}}
$$

and 0 is the empty map. Then 1 is the only unit of $V$ and $E(V)=\{0, x, y, 1\}$. Easy calculations show that the semigroup $V$ is regular and so, since the idempotents commute, $V$ is inverse. Also, since $0^{*}=0,1^{*}=1, x^{*}=x, y^{*}=y, z^{*}=w$ and $w^{*}=z, V$ is an associate inverse semigroup of itself. It can easily be verified that both conditions (A1) and (A2) are satisfied. However, since $w 1 w=w w=0 \neq w$, the semigroup $V$ is not unit regular and so it is not uniquely unit regular.

The characterization of a certain class of orthodox monoids that we obtain in section 3 uses the $\lambda$-semidirect product of a band by an inverse semigroup. We next define this product and show that the $\lambda$-semidirect product of a band with identity by an inverse monoid is a particular case of the restricted semidirect product.

Let $V$ be an inverse semigroup acting on a band $B$ by endomorphisms on the left. Then

$$
\left\{(e, v) \in B \times V: v v^{-1} e=e\right\}
$$

is an orthodox semigroup under the multiplication

$$
(e, v)(f, w)=\left({ }^{v w w^{-1} v^{-1}} e^{v} f, v w\right) .
$$

It is called the $\lambda$-semidirect product of $B$ by $V$ and it is denoted by $B *_{\lambda} V$. 
Proposition 2.7. Let $S=B *_{\lambda} V$ be a $\lambda$-semidirect product of a band with identity by an inverse monoid $V$. Then $S$ is a monoid isomorphic to a restricted semidirect product of a band with identity by an inverse monoid.

Proof. $S$ is a monoid. Let $\varepsilon$ be the identity of $B$ and $1_{V}$ be the identity of $V$. We show that $\left({ }^{1_{V}} \varepsilon, 1_{V}\right)$ is the identity of $S$. In fact, since ${ }^{1_{V} 1_{V}^{-1}}\left({ }^{1} \varepsilon\right)={ }^{1_{V} 1_{V}} \varepsilon={ }^{1_{V}} \varepsilon,\left({ }^{1_{V}} \varepsilon, 1_{V}\right) \in S$. Moreover, for each $(e, v) \in S$, we have

$$
\begin{aligned}
(e, v)\left({ }^{1_{V}} \varepsilon, 1_{V}\right) & =\left({ }^{v v^{-1}} e{ }^{v} \varepsilon, v\right)=\left({ }^{v}\left(v^{-1} e \varepsilon\right), v\right) \\
& =\left({ }^{v v^{-1}} e, v\right)=(e, v)
\end{aligned}
$$

and

$$
\begin{aligned}
\left({ }^{1} \varepsilon, 1_{V}\right)(e, v) & =\left({ }^{v v^{-1}} \varepsilon v v^{-1} e, v\right)=\left({ }^{v v^{-1}}(\varepsilon e), v\right) \\
& =\left({ }^{v v^{-1}} e, v\right)=(e, v) .
\end{aligned}
$$

Define the following subset of $S$

$$
S^{*}=\left\{\left({ }^{v v^{-1}} \varepsilon, v\right): v \in V\right\} .
$$

Then, $S^{*}$ is a subsemigroup of $S$. In fact, for every $v, w \in V,\left({ }^{v v^{-1}} \varepsilon, v\right) \in B \times V$ and $v v^{-1}\left(v^{-1} \varepsilon\right)={ }^{v v^{-1}} \varepsilon$. Moreover, for $\left({ }^{v v^{-1}} \varepsilon, v\right),\left({ }^{w w^{-1}} \varepsilon, w\right) \in S^{*}$, we have

$$
\begin{aligned}
\left({ }^{v v^{-1}} \varepsilon, v\right)\left(w w^{-1} \varepsilon, w\right) & =\left(v w w^{-1} v^{-1} v v^{-1} \varepsilon v w w^{-1} \varepsilon, v w\right) \\
& =\left(v w w^{-1}\left(v^{-1} \varepsilon \varepsilon\right), v w\right)=\left(v w w^{-1} v^{-1} \varepsilon, v w\right) \\
& =\left(v w(v w)^{-1} \varepsilon, v w\right) \in S^{*} .
\end{aligned}
$$

The semigroup $S^{*}$ is clearly inverse because the mapping $\psi: V \longrightarrow S^{*}$ defined by

$$
v \psi=\left({ }^{v v^{-1}} \varepsilon, v\right) \quad(v \in V)
$$

is easily verified to be an isomorphism.

As before, for each $(e, v) \in S$, the element $\left(v^{-1} v \varepsilon, v^{-1}\right)$ of $S^{*}$ is the least associate of $(e, v)$ in $S^{*}$.

Finally, similar calculations show that conditions (A1) and (A2) are satisfied.

So, by Corollary 2.4, $S$ is isomorphic to a restricted semidirect product of a band by an inverse semigroup.

\section{Homomorphic IMAges}

In this section we consider a more general situation in which an orthodox monoid $S$ has an inverse subsemigroup containing an associate (not necessarily a least one) of each element of $S$. These semigroups occur naturally as homomorphic images of a $\lambda$-semidirect 
product of a band with identity by an inverse monoid. The main result of this section, Theorem 3.4, provides a characterization of all such homomorphic images. In this context, the following conditions on orthodox monoids containing an inverse subsemigroup are crucial:

(H1) For each $s \in S, A(s) \cap V \neq \emptyset$;

(H2) For all $e \in E(S)$ and for all $a \in E(V)$, ea $=a e$.

Notice that such an inverse subsemigroup of $S$ is necessarily a submonoid of $S$ since by (H1) the identity of $S$ is in $V$.

We begin with two examples of such orthodox monoids.

Example 3.1. Let $B$ be a set and $A$ a proper subset of $B$ with at least two elements. Consider the following subset of the symmetric inverse semigroup on $B$

$$
S=\{\alpha \in \mathcal{I}(B): \operatorname{dom} \alpha \subset B, \operatorname{ran} \alpha \subset B \text { and }(x \in A \Leftrightarrow x \alpha \in A)\} .
$$

$S^{1}$ is clearly an inverse monoid and, hence, an orthodox submonoid of $\mathcal{I}(B)$. The subset $S^{*}:=\left\{\alpha \in S^{1}:\left.\alpha\right|_{A}\right.$ is a permutation of $\left.A\right\}$ is an inverse subsemigroup of $S^{1}$ which is not associate. In fact, if $\alpha \in S^{1}$ is such that $A \backslash \operatorname{dom} \alpha=\left\{x_{1}, x_{2}\right\}$, then $A \backslash \operatorname{ran} \alpha$ has exactly two elements, $y_{1}$ and $y_{2}$, say. So, the mappings $\alpha^{\prime}, \alpha^{\prime \prime}: \operatorname{ran} \alpha \cup\left\{y_{1}, y_{2}\right\} \rightarrow \operatorname{dom} \alpha \cup\left\{x_{1}, x_{2}\right\}$ defined by

$$
x \alpha^{\prime}=\left\{\begin{array}{cl}
x \alpha^{-1} & \text { if } x \in \operatorname{ran} \alpha \\
x_{1} & \text { if } x=y_{1} \\
x_{2} & \text { if } x=y_{2}
\end{array} \quad x \alpha^{\prime \prime}=\left\{\begin{array}{cl}
x \alpha^{-1} & \text { if } x \in \operatorname{ran} \alpha \\
x_{2} & \text { if } x=y_{1} \\
x_{1} & \text { if } x=y_{2}
\end{array}\right.\right.
$$

are two non-comparable associates of $\alpha$ in $S^{*}$. Since any other associate $\beta$ of $\alpha$ in $S^{*}$ is such that $\beta \geq \alpha^{\prime}$ or $\beta \geq \alpha^{\prime \prime}$, the least associate of $\alpha$ in $S^{*}$ doesn't exist. Clearly, (H1) and (H2) are satisfied.

Example 3.2. Consider $S=\{x \in \mathbb{R}: x \leq 2\} \backslash\{\sqrt{2}\}$ with $x y:=\min \{x, y\}$, with respect to the usual order. Then $V=] \sqrt{2}, 2]$ is an inverse subsemigroup of $S$ containing an associate of every element of $S$. However, if $x \in S \backslash V$, there is not a least associate for $x$ in $V$. Conditions (H1) and (H2) are trivially satisfied.

We proceed to characterize the orthodox monoids referred to in the beginning of this section.

Proposition 3.1. Let $S$ be an orthodox monoid with an inverse subsemigroup $V$ which satisfies conditions (H1) and (H2). Then, every homomorphic image $T$ of $S$ has an inverse subsemigroup satisfying (H1) and (H2).

Proof. Let $\varphi: S \rightarrow T$ be an epimorphism. We show that $V \varphi$ is the required inverse subsemigroup of $T$. By [[9], VII Proposition 1.2], $V \varphi$ is an inverse semigroup. Obviously $T$ 
satisfies (H1) with respect to $V \varphi$. We now show that condition (H2) holds. Let $a \in E(V \varphi)$. Then $a=u \varphi$, for some $u \in V$. Let $w=u\left(u^{2}\right)^{-1} u$. Clearly, $w$ is an idempotent of $V$. Also,

$$
w \varphi=\left[u\left(u^{2}\right)^{-1} u\right] \varphi=u \varphi\left((u \varphi)^{2}\right)^{-1} u \varphi=a\left(a^{2}\right)^{-1} a=a .
$$

Thus $a \in(E(V)) \varphi$. Now let $e \in E(T)$. By Lallement's Lemma, $e=f \varphi$ for some $f \in E(S)$. Since, by (H2), $w f=f w$, we have, by the homomorphism, ae $=e a$.

Combining Propositions 3.1 and 2.7 and Theorem 2.6, we obtain

Proposition 3.2. Let $S$ be a homomorphic image of a $\lambda$-semidirect product of a band with identity by an inverse monoid. Then $S$ is an orthodox monoid which contains an inverse subsemigroup $V$ satisfying conditions (H1) and (H2).

Proposition 3.3. Let $S$ be an orthodox monoid which contains an inverse semigroup $V$ satisfying conditions (H1) and (H2). Then $S$ is a homomorphic image of a $\lambda$-semidirect product of a band with identity by an inverse monoid.

Proof. Since $S$ is orthodox and has an identity, $E(S)$ is a band with identity $1_{S}$. Also, by (H1) there is $v \in V$ such that $1_{S} v 1_{S}=1_{S}$; thus $v=1_{S}$ and so $1_{S} \in V$. Hence the inverse subsemigroup of $S$ is a monoid.

We proceed to construct $E(S) *_{\lambda} V$. In order to do this, we define an action of $V$ on $E(S)$ by ${ }^{v} e=v e v^{-1}$, for each $e \in E(S)$ and $v \in V$. In fact,

$$
\begin{aligned}
& \text { - }{ }^{v} e f=v e f v^{-1}=v v^{-1} v e f v^{-1}=v e v^{-1} v f v^{-1}={ }^{v} e e^{v} f ; \\
& \text { - }{ }^{v w} e=(v w) e(v w)^{-1}=v w e w^{-1} v^{-1}=v\left({ }^{w} e\right) v^{-1}={ }^{v}\left({ }^{w} e\right) ; \\
& \text { - }{ }^{1_{V}} e=1_{V} e 1_{v}^{-1}=e .
\end{aligned}
$$

Notice that, by (H2), this action is such that

$$
v v^{-1} e=v v^{-1} e\left(v v^{-1}\right)^{-1}=e v v^{-1}\left(v v^{-1}\right)^{-1}=e v v^{-1}
$$

and similarly, ${ }^{v v^{-1}} e=v v^{-1} e$.

Let $U=E(S) *_{\lambda} V$ be a $\lambda$-semidirect product of $E(S)$ by $V$, with respect to the action defined above. Then

$$
U=\left\{(e, v) \in E(S) \times V: v^{-1} e=e\right\}=\left\{(e, v) \in E(S) \times V: v v^{-1} e=e\right\}
$$

and

$$
\begin{aligned}
(e, v)(f, w) & =\left(v w w^{-1} v^{-1} e^{v} f, v w\right)=\left(v w w^{-1} v^{-1} e v f v^{-1}, v w\right) \\
& =\left(e v w w^{-1} v^{-1} v f v^{-1}, v w\right)=\left(e v v^{-1} v w w^{-1} f v^{-1}, v w\right) \\
& =\left(e v w w^{-1} f v^{-1}, v w\right)=\left(e v f v^{-1}, v w\right)=\left(e^{v} f, v w\right) .
\end{aligned}
$$

Notice that $U$ is a subsemigroup of the semidirect product $E(S) * V$. 
Next define a mapping $\psi: U \rightarrow S$ by $(e, v) \psi=e v$. For any $(e, v),(f, w) \in U$,

$$
\begin{aligned}
((e, v)(f, w)) \psi & =\left(e v f v^{-1}, v w\right) \psi=e v f v^{-1} v w \\
& =e v v^{-1} v f w=e v f w \\
& =(e, v) \psi(f, w) \psi
\end{aligned}
$$

Thus $\psi$ is a homomorphism. It is also surjective. In fact, if $s \in S$ then by (H1) $s=s v s=$ $s v v^{-1} v s$, for some $v \in V$. Since $v v^{-1} \in E(V)$ and $v s \in E(S)$, (H2) gives

$$
s=s v s v v^{-1}=s v v^{-1},
$$

where $s v \in E(S)$ and $v^{-1} \in V$. Since

$$
v^{-1} v s v=v^{-1} v s v v^{-1} v=v^{-1} v s v=s v v^{-1} v=s v,
$$

it follows that $\left(s v, v^{-1}\right) \in U$. Finally, (5) gives

$$
s=s v v^{-1}=\left(s v, v^{-1}\right) \psi,
$$

whence $\psi$ is surjective. The result follows.

Combining Propositions 3.2 e 3.3 we obtain the following characterization.

Theorem 3.4. Let $S$ be a homomorphic image of a $\lambda$-semidirect product of a band with identity by an inverse monoid. Then $S$ is an orthodox monoid containing an inverse subsemigroup $V$ which satisfies

(H1) For each $s \in S, A(s) \cap V \neq \emptyset$;

(H2) For all $e \in E(S)$ and for all $a \in E(V)$, ea $=$ ae.

Conversely, every such semigroup can be constructed this way.

We observe that [[10] Theorem 1.4] is a corollary of Theorem 3.4 above: if $S$ is a unit regular orthodox semigroup and $U$ is its group of units, then (H2) is trivially satisfied and (H1) follows from the fact that $S$ is unit regular. So, $\varphi: E(S) *_{\lambda} U \rightarrow S$ defined by $(e, u) \varphi=e u$ is an epimorphism. Since $E\left(E(S) *_{\lambda} U\right)=E(S) \times\left\{1_{S}\right\}, S$ is an idempotentseparating homomorphic image of a $\lambda$-semidirect product of $E(S)$ by $U$. [[10] Theorem 1.4] now follows from Proposition 2.7.

Not every orthodox monoid $S$ with an inverse semigroup satisfying conditions (H1) and (H2) is unit regular. In fact, although the orthodox monoid $S^{1}$ defined in Example 3.1 has an inverse subsemigroup containing an associate of each of its elements and satisfies

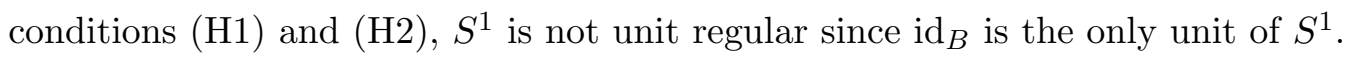




\section{A particular Case: F-Regular Semigroups}

$F$-regular semigroups were first described by Edwards in [6] where it is proven that $F$-regular semigroups are orthodox monoids. Further characterizations of $F$-regular semigroups are provided in [7] and [2].

In this section we prove that $F$-regular semigroups have an inverse subsemigroup $S$, satisfying conditions (H1) and (H2) stated at the beginning of Section 3, which is associate when $S$ is finite. If $S$ is not finite, this subsemigroup is not necessarily associate, as it is shown by Example 4.1.

Let $S$ be an $F$-regular semigroup and $\sigma$ the least group congruence on $S$. For each $a \in S$, we denote the greatest element of $a \sigma$ by $a^{\top \top}$ and the greatest element of $(a \sigma)^{-1}$ by $a^{\top}$. By [[7], Corollary 2.5], $a^{\top}$ is an associate of $a$ in $S$ (in fact, the greatest one).

Proposition 4.1. Let $S$ be an F-regular semigroup and $W$ the subsemigroup of $S$ generated by $\left\{a^{\top \top}: a \in S\right\}$. Then $W$ is a regular submonoid of $S$ in which each element lies beneath a generator of $W$.

Proof. Since $S$ is $F$-regular, it follows by [[7], Corollary 2.4] that $S$ has an identity $1_{S}$, say; since $1_{S}=1_{S}^{\top \top}$, it follows that $W$ is a submonoid of $S$. By [[7], Theorem 2.2], for each $a \in W$, the element $a^{\top}$ is an associate of $a$ and, since $a^{\top}=\left(a^{\top}\right)^{\top \top}$ by [[7], Proposition 4.1(iii)], it follows that $W$ is a regular submonoid of $S$. Finally, by [[7], Proposition 4.1(i)], $a \leq a^{\top \top}$, for each $a \in W$.

Lemma 4.2. Let $S$ be an F-regular semigroup. Then, for all idempotent $e \in S$ and all $s_{1}^{\top \top}, s_{2}^{\top \top}, \ldots, s_{n}^{\top \top} \in W \quad(n \in \mathbb{N})$, we have

$$
s_{1}^{\top \top} s_{2}^{\top \top} \cdots s_{n}^{\top \top} s_{n}^{\top} \cdots s_{2}^{\top} s_{1}^{\top} \cdot e=e \cdot s_{1}^{\top \top} s_{2}^{\top \top} \cdots s_{n}^{\top \top} s_{n}^{\top} \cdots s_{2}^{\top} s_{1}^{\top} .
$$

Proof. We prove this lemma using induction on the number of factors in $s_{1}^{\top \top}, s_{2}^{\top \top}, \ldots, s_{n}^{\top \top}$.

For $n=1$, we have, for all $e \in E(S)$, by [[7], Propositions 4.1(vii) and 4.2(viii)], that

$$
\begin{aligned}
s_{1}^{\top \top} s_{1}^{\top} \cdot e & =s_{1}^{\top \top} \cdot s_{1}^{\top} e=s_{1}^{\top \top} \cdot s_{1}^{\top} e s_{1}^{\top \top} s_{1}^{\top} \\
& =s_{1}^{\top \top} s_{1}^{\top} e s_{1}^{\top \top} \cdot s_{1}^{\top}=e s_{1}^{\top \top} s_{1}^{\top}
\end{aligned}
$$


Now, let $k \in \mathbb{N}, s_{1}, s_{2}, \ldots, s_{k}$ be $k$ arbitrarily chosen elements in $S$ and $e \in E(S)$. Consider the elements $s_{1}^{\top \top}, s_{2}^{\top \top}, \ldots, s_{k}^{\top \top} \in W$ and suppose that the result holds for $k-1$. We have

$$
\begin{aligned}
s_{1}^{\top \top} s_{2}^{\top \top} \cdots s_{k-1}^{\top \top} s_{k}^{\top \top} s_{k}^{\top} s_{k-1}^{\top} \cdots s_{2}^{\top} s_{1}^{\top} e=s_{1}^{\top \top} s_{2}^{\top \top} \cdots s_{k-1}^{\top \top} s_{k}^{\top \top} s_{k}^{\top} s_{k-1}^{\top} \cdots s_{2}^{\top} s_{1}^{\top} e s_{1}^{\top \top} s_{1}^{\top} & {[[7], \text { Proposition 4.1(vii)] }} \\
= & s_{1}^{\top \top} \cdot s_{2}^{\top \top} \cdots s_{k-1}^{\top \top} s_{k}^{\top \top} s_{k}^{\top} s_{k-1}^{\top} \cdots s_{2}^{\top}\left(s_{1}^{\top} e s_{1}^{\top \top}\right) \cdot s_{1}^{\top} \\
& =s_{1}^{\top \top}\left(s_{1}^{\top} e s_{1}^{\top \top}\right) s_{2}^{\top \top} \cdots s_{k-1}^{\top \top} s_{k}^{\top \top} s_{k}^{\top} s_{k-1}^{\top} \cdots s_{2}^{\top} s_{1}^{\top} \\
& {[\text { induction hypothesis] }} \\
& =e s_{1}^{\top \top} s_{2}^{\top \top} \cdots s_{k-1}^{\top \top} s_{k}^{\top \top} s_{k}^{\top} s_{k-1}^{\top} \cdots s_{2}^{\top} s_{1}^{\top} .
\end{aligned}
$$

The result follows by induction.

Lemma 4.3. Let $S$ be an F-regular semigroup and $W$ the regular submonoid generated by $\left\{s^{\top \top}: s \in S\right\}$. Then each idempotent $s_{1}^{\top \top} s_{2}^{\top \top} \cdots s_{k}^{\top \top}$ of $W(k \in \mathbb{N})$ can be written both as $s_{1}^{\top \top} s_{2}^{\top \top} \cdots s_{k}^{\top \top} s_{k}^{\top} \cdots s_{2}^{\top} s_{1}^{\top}$ and $s_{k}^{\top} \cdots s_{2}^{\top} s_{1}^{\top} s_{1}^{\top \top} s_{2}^{\top \top} \cdots s_{k}^{\top \top}$.

Proof. We prove this lemma using induction on the number of factors in $s_{1}^{\top \top} s_{2}^{\top \top} \cdots s_{k}^{\top \top}$.

For $k=1$,

$$
s_{1}^{\top \top} \in E(S) \Rightarrow s_{1} \sigma=E(S) \Rightarrow s_{1}^{\top \top}=1_{S} \Rightarrow s_{1}^{\top}=1_{S}
$$

and so, $s_{1}^{\top \top}=s_{1}^{\top \top} s_{1}^{\top}$.

For $k=2$, we have

$$
s_{1}^{\top \top} s_{2}^{\top \top} \in E(S) \Rightarrow s_{1}^{\top \top} s_{2}^{\top \top}=s_{1}^{\top \top} s_{1}^{\top}=s_{1}^{\top \top} s_{1}^{\top} s_{1}^{\top \top} s_{1}^{\top}=s_{1}^{\top \top} s_{2}^{\top \top} s_{2}^{\top} s_{1}^{\top} .
$$

Now let $k \in \mathbb{N}$ and let $s_{1}^{\top \top} s_{2}^{\top \top} \cdots s_{k}^{\top \top}$ and idempotent of $S$. Suppose that the result holds for $k-1$. Our argument to show that the result is also valid for $k$ uses the fact that it holds for $k=3$. So we start by showing that

$$
s_{1}^{\top \top} s_{2}^{\top \top} s_{3}^{\top \top}=s_{1}^{\top \top} s_{2}^{\top \top} s_{3}^{\top \top} s_{3}^{\top} s_{2}^{\top} s_{1}^{\top}=s_{3}^{\top} s_{2}^{\top} s_{1}^{\top} s_{1}^{\top \top} s_{2}^{\top \top} s_{3}^{\top \top} .
$$

Since $s_{1}^{\top \top} s_{2}^{\top \top} s_{3}^{\top \top} \in E(S)$, we have

$$
\begin{array}{rlrl}
s_{1}^{\top \top} s_{2}^{\top \top} s_{3}^{\top \top} & =s_{1}^{\top \top} s_{2}^{\top \top}\left(s_{1} s_{2}\right)^{\top} & \\
& =s_{1}^{\top \top} s_{2}^{\top \top} s_{2}^{\top} s_{1}^{\top} & & {[[2], \text { Lemma } 1(i v)]} \\
& =s_{1}^{\top \top} s_{2}^{\top \top} s_{3}^{\top \top} s_{3}^{\top} s_{2}^{\top} s_{1}^{\top} . & {[[7], \text { Proposition 4.2(viii) }]}
\end{array}
$$


Now suppose that $s_{1}^{\top \top} s_{2}^{\top \top} \cdots s_{k}^{\top \top}$ is an idempotent of $S$. We have

$$
\begin{aligned}
s_{1}^{\top \top} s_{2}^{\top \top} \cdots s_{k-2}^{\top \top} s_{k-1}^{\top \top} s_{k}^{\top \top}=\left(s_{1}^{\top \top} s_{2}^{\top \top} \cdots s_{k-2}^{\top \top}\right)\left(s_{k-1}^{\top \top} s_{k}^{\top \top}\right) & \\
= & s_{1}^{\top \top} s_{2}^{\top \top} \cdots s_{k-2}^{\top \top}\left(\left(s_{1} s_{2} \cdots s_{k-2}\right)^{\top}\left(s_{1} s_{2} \cdots s_{k-2}\right)^{\top \top}\right) s_{k-1}^{\top \top} s_{k}^{\top \top} \\
= & \left(s_{1}^{\top \top} s_{2}^{\top \top} \cdots s_{k-2}^{\top \top}\left(s_{1} s_{2} \cdots s_{k-2}\right)^{\top}\right)\left(\left(s_{1} s_{2} \cdots s_{k-2}\right)^{\top \top} s_{k-1}^{\top \top} s_{k}^{\top \top}\right) \\
= & s_{1}^{\top \top} s_{2}^{\top \top} \cdots s_{k-2}^{\top \top}\left(s_{1} s_{2} \cdots s_{k-2}\right)^{\top}\left(s_{1} s_{2} \cdots s_{k-2}\right)^{\top \top} s_{k-2}^{\top} \cdots s_{2}^{\top} s_{1}^{\top} . \\
& \cdot\left(s_{1} s_{2} \cdots s_{k-2}\right)^{\top \top} s_{k-1}^{\top \top} s_{k}^{\top \top} s_{k}^{\top} s_{k-1}^{\top}\left(s_{1} s_{2} \cdots s_{k-2}\right)^{\top} \\
= & \left(s_{1}^{\top \top} s_{2}^{\top \top} \cdots s_{k-2}^{\top \top}\left(s_{1} s_{2} \cdots s_{k-2}\right)^{\top}\left(s_{1} s_{2} \cdots s_{k-2}\right)^{\top \top}\right) . \\
& \quad \cdot\left(s_{k-2}^{\top} \cdots s_{2}^{\top} s_{1}^{\top}\left(s_{1} s_{2} \cdots s_{k-2}\right)^{\top \top}\right) \cdot\left(s_{k-1}^{\top \top} s_{k}^{\top \top} s_{k}^{\top} s_{k-1}^{\top}\left(s_{1} s_{2} \cdots s_{k-2}\right)^{\top}\right) \\
= & s_{1}^{\top \top} s_{2}^{\top \top} \cdots s_{k-2}^{\top \top} \cdot\left(s_{k-2}^{\top} \cdots s_{2}^{\top} s_{1}^{\top}\left(s_{1} s_{2} \cdots s_{k-2}\right)^{\top \top}\right) . \\
= & \cdot\left(s_{k-1}^{\top \top} s_{k}^{\top \top} s_{k}^{\top} s_{k-1}^{\top}\right)\left(s_{1} s_{2} \cdots s_{k-2}\right)^{\top} \\
= & s_{1}^{\top \top} s_{2}^{\top \top} \cdots s_{k-2}^{\top \top} s_{k-1}^{\top \top} s_{k}^{\top \top} s_{k}^{\top} s_{k-1}^{\top}\left(s_{k-2}^{\top} \cdots s_{2}^{\top} s_{1}^{\top}\left(s_{1} s_{2} \cdots s_{k-2}\right)^{\top \top}\left(s_{1} s_{2} \cdots s_{k-2}\right)^{\top \top}\right) \\
= & \left(s_{1} s_{2} \cdots s_{k-2}\right)^{\top \top}\left(s_{1} s_{2} \cdots s_{k-2}\right)^{\top \top} s_{1}^{\top \top} s_{2}^{\top \top} \cdots s_{k-2}^{\top \top} s_{k-1}^{\top \top} s_{k}^{\top \top} s_{k}^{\top} s_{k-1}^{\top} s_{k-2}^{\top} \cdots s_{2}^{\top} s_{1}^{\top} \\
= & s_{1}^{\top \top} s_{2}^{\top \top} \cdots s_{k-2}^{\top \top} s_{k-1}^{\top \top} s_{k}^{\top \top} s_{k}^{\top} s_{k-1}^{\top} s_{k-2}^{\top} \cdots s_{2}^{\top} s_{1}^{\top} .
\end{aligned}
$$

The result follows by induction.

Similarly, we can prove that

$$
s_{1}^{\top \top} s_{2}^{\top \top} \cdots s_{k}^{\top \top}=s_{k}^{\top} \cdots s_{2}^{\top} s_{1}^{\top} s_{1}^{\top \top} s_{2}^{\top \top} \cdots s_{k}^{\top \top} .
$$

Proposition 4.4. Let $S$ be an F-regular semigroup and $W$ the submonoid generated by the set $\left\{s^{\top \top}: s \in S\right\}$. Then $W$ satisfies the following properties:

(H1) For each $s \in S A(s) \cap W \neq \emptyset$;

(H2) For each $e \in E(S)$ and for every $a \in E(W)$, ea $=$ ae.

Proof. (H1) Let $s \in S$. Since the semigroup $S$ is $F$-regular, it follows by [[7], Theorem $2.2]$ that $s=s s^{\top} s$. By [[7], Proposition 4.1(iii)], $s^{\top}=s^{\top \top \top} \in W$ and the result follows.

(H2) This is an immediate consequence of Lemmas 4.2 and 4.3.

Corollary 4.5. In an F-regular semigroup, the submonoid $W$ generated by the set $\left\{s^{\top \top}\right.$ : $s \in S\}$ is an $F$-inverse semigroup.

Proof. By Proposition 4.1, the monoid $W$ is regular. By Proposition 4.4 the idempotents of $W$ commute. Thus the semigroup $W$ is inverse. Also, by Proposition 4.1, each $x \in W$ is such that $x \leq x^{\top \top} \in W$. Hence, by [[7], Theorem 5.3(ii)], $W$ is an $F$-semigroup and the result follows. 
Corollary 4.6. Every F-regular semigroup is an orthodox monoid that has an inverse subsemigroup satisfying conditions ( $\mathrm{H} 1)$ and $(\mathrm{H} 2)$.

Proposition 4.7. For a finite F-regular semigroup, the inverse subsemigroup $W$ is associate.

Proof. Let $s \in S$ and $u, v \in A(s) \cap W$. By [[2], Lemma 1(ii)], $u=u s^{\top \top} u$ and $v=v s^{\top \top} v$ and so the elements $u s^{\top \top}, s^{\top \top} v$ are idempotents of $W$. Thus $u s^{\top \top} v \in W$ lies beneath both $u$ and $v$. We have, by [[2], Lemma 1(i)], $u s^{\top \top} s^{\top}=u=s^{\top} s^{\top \top} u$ and $v s^{\top \top} s^{\top}=v=$ $s^{\top} s^{\top \top} v$. Then, since $s u \in E(S)$ and $s^{\top \top} v \in E(W)$, it follows from (H2) that

$$
\text { sus }^{\top \top} v s=s^{\top \top} \text { vsus }=s^{\top \top} v s=s^{\top \top} v s s^{\top} s=s s^{\top} s^{\top \top} v s=s v s=s .
$$

So, the element $u s^{\top \top} v \in A(s) \cap W$ is such that $u s^{\top \top} v \leq u, v$. Thus, if $S$ is finite, the inverse subsemigroup $W$ of $S$ is associate.

The following example shows that if an $F$-regular semigroup $S$ is not finite then the subsemigroup $W$ of $S$ is not necessarily associate.

Example 4.1. Let $E$ be the infinite chain

$$
\begin{aligned}
& \varepsilon \\
& \varepsilon_{1} \\
& \varepsilon_{2} \\
& \cdot \\
& \cdot \\
& \bullet \\
& \cdot \\
& \cdot \\
& \cdot \\
& \cdot \\
& \bullet
\end{aligned}
$$

and $F_{A G}(X)$ be the free abelian group on the infinite set $X=\left\{x_{1}, x_{2}, \ldots, x_{n}, \ldots\right\}$. Consider the direct product $E \times F_{A G}(X)$ and let $S$ be the inverse submonoid of $F_{A G}(X)$ generated by the set $M=\{(0,1),(\varepsilon, 1)\} \cup\left\{\left(\varepsilon_{n}, x_{n}\right) \mid n \in \mathbb{N}\right\}$. We claim that $S$ is an infinite $F$ regular semigroup, for which the inverse subsemigroup $W$ is not associate. The binary relation $\sigma$ on $S$ given by the equality of the second component is a group congruence on $S$. If $x=x_{i_{1}}^{n_{1}} x_{i_{2}}^{n_{2}} \ldots x_{i_{k}}^{n_{k}}$, with $n_{1}, n_{2}, \ldots, n_{k} \in \mathbb{Z}, i_{1}, i_{2}, \ldots, i_{k} \in \mathbb{N}$ and $i_{1}<i_{2}<\ldots<i_{k}$, and $\left(\varepsilon_{j}, x\right) \in S$ then $j \geq i_{k}$ and $\left(\varepsilon_{j}, x\right)=\left(\varepsilon_{j}, x_{j}\right)\left(\varepsilon_{j}, x_{j}^{-1}\right)\left(\varepsilon_{i_{k}}, x\right)$. Thus, $\left[\left(\varepsilon_{j}, x\right)\right]=$ $\{(0, x)\} \cup\left\{\left(\varepsilon_{i}, x\right) \mid i \geq i_{k}\right\}$, giving $\left(\varepsilon_{j}, x\right)^{\top \top}=\left(\varepsilon_{i_{k}}, x\right)$. Clearly, $(0,1)^{\top \top}=(\varepsilon, 1)^{\top \top}=(\varepsilon, 1)$, $\left(0, x_{n}\right)^{\top \top}=\left(0, x_{n}\right)$ and $\left(\varepsilon, x_{n}\right)^{\top \top}=\left(\varepsilon, x_{n}\right)$, for all $n \in \mathbb{N}$. Therefore, the monoid $S$ is $F$-inverse, with $W=S \backslash\left\{(0, x): x \in F_{A G}(X)\right\}$. However, since the associate elements of $(0,1)$ in $W$ are precisely the elements $\left(\varepsilon_{n}, 1\right)(n \in \mathbb{N})$ and $(\varepsilon, 1)$, there is no least one in $W$ and so the inverse subsemigroup $W$ of $S$ is not associate. 
Finally, we observe that, by [[7], Theorem 6.4], F-regular $T$-semigroups are uniquely unit regular. So, it follows from the observation after Example 2.2 that $F$-regular $T$ semigroups are orthodox monoids that contain an inverse subsemigroup satisfying (A1) and (A2) stated in Section 2. Notice that in Example 4.1, since $W$ is not associate, it also follows that $S$ is not a $T$-semigroup.

\section{REFERENCES}

[1] B. Billhardt. On a semigroup theoretic generalization of the Kalužnin Krasner Theorem and normal extensions of inverse semigroups. Semigroup Forum 44 (1992) 364-372

[2] B. Billhardt. A representation for F-regular semigroups. Semigroup Forum 70 (2005) 243-251

[3] B. Billhardt, E. Giraldes, P. Marques-Smith and P. Mendes Martins. Associate inverse subsemigroups of regular semigroups. Semigroup Forum 79 (2009) 101-118

[4] T. S. Blyth, Emília Giraldes and M. Paula O. Marques-Smith. Associate subgroups of orthodox semigroups. Glasgow Math J. 36 (1994) 163-171

[5] T.S. Blyth and R. McFadden. Unit orthodox semigroups. Glasgow Math. J. 24 (1983) 39-42

[6] C.C. Edwards. F-regular and F-orthodox semigroups. Semigroup Forum 19 (1980) 331-345

[7] E. Giraldes, P. Marques-Smith and H. Mitsch. F-regular semigroups. J. Algebra 274 (2004) 491-510

[8] K.R. Goodearl. von Neumann regular rings. Pitman (1979)

[9] P.A. Grillet. Semigroups: an introduction to the structure theory. Marcell Dekker, New York (1995)

[10] R. McFadden. Unit-regular orthodox semigroups. Glasgow Math. J. 25 (1984) 229-240

[11] M. Petrich. Introduction to Semigroups. Charles E. Merrill Publishing Co.; A. Bell and Howell Company. Columbus (1973)

$\begin{array}{llll}\text { Universität Kassel } & \text { U.T.A.D. } & \text { Universidade do Minho } & \text { Universidade do Minho } \\ \text { FB - 17 Mathematik } & \text { CM-UTAD } & \text { Centro de Matemática } & \text { Centro de Matemática } \\ \text { D-34127 Kassel, Germany } & \text { 5000 Vila Real, Portugal } & \text { 4710-057 Braga, Portugal } & \text { 4710-057 Braga, Portugal } \\ \text { billhardt@uni-kassel.de } & \text { egs@utad.pt } & \text { psmith@math.uminho.pt } & \text { pmendes@math.uminho.pt }\end{array}$

